\title{
Democracy and polarization in the National Assembly of the Republic of Korea
}

\author{
Jonghoon Kim and Seung Ki Baek \\ Department of Physics, Pukyong National University, Busan 48513, Korea
}

\begin{abstract}
The median-voter hypothesis predicts convergence of party platforms across a one-dimensional political spectrum during majoritarian elections. Assuming that the convergence is reflected in legislative activity, we study the time evolution of political polarization in the National Assembly of the Republic of Korea for the past 70 years. By projecting the correlation of lawmakers onto the first principal axis, we observe a high degree of polarization from the early 1960's to the late 1980's before democratization. As predicted by the hypothesis, it showed a sharp decrease when party politics were revived in 1987. Since then, the political landscape has become more and more multi-dimensional under the action of party politics, which invalidates the assumption behind the hypothesis. For comparison, we also analyze co-sponsorship in the United States House of Representatives from 1979 to 2020, whose correlation matrix has been constantly high-dimensional throughout the observation period. Our analysis suggests a pattern of polarization evolving with democratic development, from which we can argue the power and the limitation of the median-voter hypothesis as an explanation of real politics.
\end{abstract}

Keywords: Principal-component analysis, Co-sponsorship, Median-voter hypothesis, Political polarization

\footnotetext{
* seungki@pknu.ac.kr; Fax: +82-51-629-5549
} 


\section{INTRODUCTION}

Democracy is noisy. Through party politics, it invites the public into conflicts that were once private so that the conflicts are socialized as public affairs [1, 2]. A conflict of great social importance becomes a cleavage around which parties are positioned, and the parties compete for votes by representing different views on the cleavage. Therefore, polarization of parties is an inherent and inevitable feature of democracy, despite growing concern about its negative consequences [3, 4]. At the same time, the median-voter hypothesis (MVH) argues that two parties will converge to the same position across a one-dimensional political spectrum as long as voters always vote with single-peaked preferences [5, 6]: To be more specific, let us imagine a number of voters, each of whom has his or her own political position as a real number and votes for a party whose position is the closest to his or her own. A party has to obtain more votes than the other parties to win the election, and if two parties play this zero-sum game, the Nash equilibrium is reached when both converge to the median voter's position. A rough sketch of party politics would be an interplay between these two forces of polarization and convergence.

The empirical evidence for the MVH is mixed, and the main reason is that the political privacy in the secret-ballot system poses an obstacle to its direct verification. One way to get around the difficulty is to assume that the median voter is effectively equal to a voter with the median income (or the median tax price) [7-10]. If this is the case, the MVH provides testable predictions: For example, a more unequal society is predicted to choose greater redistribution because the median voter will be relatively poorer in a more unequal society when compared with the mean income [10]. As an alternative indirect measure, one can compare the dispersion in expenditures of local governments: An early finding was that local governments with a single party showed a greater coefficient of variation than those with two parties, suggesting that moderate policies tend to be adopted as a result of political competition [11, 12]. Still, whether the MVH outperforms the existing models mostly based on mean incomes remains in doubt, and a number of studies present statistical results against the MVH $[13-15]$.

In this work, we wish to examine the dynamics of party politics by looking at the cosponsorship in the National Assembly of Korea for the past 70 years. Co-sponsorship has been used to analyze collaboration patterns among lawmakers [16]: In the United States, co- 
sponsorship is known to exhibit a lower degree of polarization than roll-call voting because successful legislation requires a supermajority in the presence of bicameralism, the filibuster, and the presidential veto [17, 18]. However, this work is relatively free from such a risk of underestimation because the immediate goal of co-sponsorship in Korea is not to pass the bill but to introduce it officially to the Assembly: It needs only 10 lawmakers, and the bill usually expresses its own idea most clearly at this stage before undergoing revisions to seek a compromise. Indeed, co-sponsorship analyses of the National Assembly of Korea have detected strong partisanship [19, 20]. We also stress that we are discussing how lawmakers perceive the party politics as a whole: If we move our focus to each instance of congressional voting, it will usually be explained by one or two dimensions [21].

Our main finding is that the dynamics of party politics tends to contradict the assumption of one dimensionality behind the MVH. We suggest that a better understanding would be to consider an inverse proposition of the MVH, i.e., predicting the lack of convergence in the absence of party politics. In addition, we propose a scenario of how political polarization would evolve in the course of democratic development. This work is organized as follows: The next section discusses our methods, including data curation and statistical observables. We present the result in Section III and discuss its implications in Section IV. We then conclude this work in Section V.

\section{METHOD}

\section{A. Data}

We have collected the bill data from 1950 to 2019 through Open Data Portal (http: //www.data.go.kr). For each bill, we have its date of motion, title, chief author, and cosponsors. The first National Assembly (May 311948 - May 30 1950) has been excluded from our analysis because it was a constituent assembly with no co-sponsored bills. We also exclude the years 1962, 1971, 1979, and 1992 when no or few bills were sponsored. See Table \ for details of the data.

To quantify the level of democracy, we use the Freedom in the World reports from 1973 to 2020 by Freedom House (https://freedomhouse.org). Those reports have two indices on a scale from 1 (most free) and 7 (least free): One measures political rights, and the other 
addresses civil liberties.

For comparison, we have also collected co-sponsorship data in the United States House of Representatives from http://www.congress.gov. The period ranges from 1979 to 2020.

\section{B. Analysis}

To examine the data from the viewpoint of the MVH, we have used principal component analysis (PCA). Let us consider $N$ data points, each of which is $M$-dimensional. For the co-sponsorship data, $N$ and $M$ are the number of lawmakers and the number of bills, respectively, for a given period. The whole data can then be represented by an $M \times N$ matrix $R$, and a column vector $\left[R_{1 n}, \ldots, R_{M n}\right]^{\top}$ denotes the $n$th data point, where $\mathrm{T}$ means transpose. We set $R_{m n}=1$ if the $n$th lawmaker sponsored the $m$ th bill, and $R_{m n}=0$ otherwise. After removing all-zero rows and columns, we work with a standardized matrix:

$$
X=\left(\begin{array}{ccc}
\frac{R_{11}-\mu_{1}}{s_{1}} & \ldots & \frac{R_{1 N}-\mu_{N}}{s_{N}} \\
\vdots & \ddots & \vdots \\
\frac{R_{M 1}-\mu_{1}}{s_{1}} & \ldots & \frac{R_{M N}-\mu_{N}}{s_{N}}
\end{array}\right)
$$

where $\mu_{n} \equiv M^{-1} \sum_{m} R_{m n}$ and $s_{n}=\sqrt{\sum_{m}\left(R_{m n}-\mu_{n}\right)^{2} /(M-1)}$ are the sample mean and standard deviation of the $n$th column vector, respectively. The next step is to construct a correlation matrix:

$$
\begin{aligned}
& Q=\frac{1}{M-1} X^{\top} X \\
& =\frac{1}{M-1}\left(\begin{array}{ccc}
X_{11} & \ldots & X_{M 1} \\
\vdots & \ddots & \vdots \\
X_{1 N} & \vdots & X_{M N}
\end{array}\right)\left(\begin{array}{ccc}
X_{11} & \ldots & X_{1 N} \\
\vdots & \ddots & \vdots \\
X_{M 1} & \vdots & X_{M N}
\end{array}\right)
\end{aligned}
$$

whose element $Q_{i j}$ is the correlation between the $i$ th and the $j$ th data points:

$$
\begin{aligned}
Q_{i j} & =\frac{1}{M-1} \sum_{m=1}^{M} X_{m i} X_{m j} \\
& =\frac{1}{M-1} \sum_{m=1}^{M}\left(\frac{R_{m i}-\mu_{i}}{s_{i}}\right)\left(\frac{R_{m j}-\mu_{j}}{s_{j}}\right) .
\end{aligned}
$$

Note that $Q_{i j}$ takes a value from $[-1: 1]$ with $Q_{i i}=1$. If $Q_{i j}$ is close to one, lawmakers $i$ and $j$ have highly correlated co-sponsorship behavior. If $Q_{i j}$ is negative, on the other 
TABLE I. Summary of data used in this work. The first column means the session number of the Assembly, and the second and the third columns show the dates of establishment and disbandment, respectively. The fourth column shows the number of seats, and the figures in the parentheses mean the numbers of lawmakers awarded through proportional representation or through recommendation of the president (marked by $*$ ). Single-member constituencies comprise the other seats in most cases, but we have a few exceptions of the medium constituency system as noted in the last column. The fifth column shows the number of collected bills. For the 20th Assembly, we use the bill data in Ref. [20], which was collected up to June 3, 2019 (†). The Assembly has been unicameral except for the 5th session.

\begin{tabular}{|c|c|c|c|c|c|}
\hline session \# & established & disbanded & $\neq$ of sea & \# of bills & misc. \\
\hline 1 & $5 / 31 / 1948$ & $5 / 30 / 1950$ & $200(0)$ & $\mathrm{N} / \mathrm{A}$ & \\
\hline 2 & $5 / 31 / 1950$ & $5 / 30 / 1954$ & $210(0)$ & 674 & \\
\hline 3 & $5 / 31 / 1954$ & $5 / 30 / 1958$ & $203(0)$ & 625 & \\
\hline 4 & $5 / 31 / 1958$ & $7 / 28 / 1960$ & $233(0)$ & 275 & \\
\hline 5 & $7 / 29 / 1960$ & $12 / 16 / 1963$ & $291(0)$ & 327 & bicameral \\
\hline 6 & $12 / 17 / 1963$ & $6 / 30 / 1967$ & $175(44)$ & 538 & \\
\hline 7 & $7 / 1 / 1967$ & $6 / 30 / 1971$ & $175(44)$ & 238 & \\
\hline 8 & $7 / 1 / 1971$ & 10/17/1972 & $204(51)$ & 66 & \\
\hline 9 & $3 / 12 / 1973$ & $3 / 11 / 1979$ & $219\left(73^{*}\right)$ & 221 & medium \\
\hline 10 & $3 / 12 / 1979$ & $10 / 27 / 1980$ & $231\left(77^{*}\right)$ & 127 & medium \\
\hline 11 & $4 / 11 / 1981$ & $4 / 10 / 1985$ & $276(92)$ & 266 & medium \\
\hline 12 & $4 / 11 / 1985$ & $5 / 29 / 1988$ & $276(92)$ & 304 & medium \\
\hline 13 & $5 / 30 / 1988$ & $5 / 29 / 1992$ & $299(75)$ & 683 & \\
\hline 14 & $5 / 30 / 1992$ & $5 / 29 / 1996$ & $299(62)$ & 427 & \\
\hline 15 & $5 / 30 / 1996$ & $5 / 29 / 2000$ & $299(46)$ & 1021 & \\
\hline 16 & $5 / 30 / 2000$ & $5 / 29 / 2004$ & $273(46)$ & 1890 & \\
\hline 17 & $5 / 30 / 2004$ & $5 / 29 / 2008$ & $299(56)$ & 6106 & \\
\hline 18 & $5 / 30 / 2008$ & $5 / 29 / 2012$ & $299(54)$ & 11564 & \\
\hline 19 & $5 / 30 / 2012$ & $5 / 29 / 2016$ & $300(54)$ & 15806 & \\
\hline 20 & $5 / 30 / 2016$ & $5 / 29 / 2020$ & $300(47)$ & $20967^{\dagger}$ & \\
\hline
\end{tabular}


hand, the lawmakers are anti-correlated, meaning that they usually do not work together. We diagonalize the correlation matrix $Q$, e.g., by using the singular-value decomposition, and take the principal eigenvector corresponding to the largest eigenvalue. This gives a one-dimensional representation of co-sponsorship among lawmakers, and the validity of this dimensionality reduction is measured by the proportion of variance explained by the principal component. If the Assembly is highly polarized, we will find two clusters of lawmakers on opposite sides of the axis, and the distance between the clusters will explain most of the variance in the data.

\section{Observables}

To quantify political polarization, we compute the bimodality coefficient [22, 23]:

$$
\beta=\frac{\gamma^{2}+1}{\kappa},
$$

where $\gamma$ is the skewness and $\kappa$ is the kurtosis of the distribution. The rationale behind this quantity is that a bimodal distribution often has asymmetric peaks and light tails, both of which increase the value of $\beta$. For the normal distribution, we get $\beta=1 / 3$ whereas the maximum value of $\beta=1$ is obtained for the bi-delta distribution. The uniform distribution with $\beta=5 / 9$ can be regarded as a borderline between unimodal and bimodal ones. For $N$ samples on the principal axis, the formula should be rewritten as

$$
b=\frac{g^{2}+1}{k+\frac{3(N-1)^{2}}{(N-2)(N-3)}}
$$

where $g$ is the sample skewness and $k$ is the sample excess kurtosis.

Another observable in PCA is the proportion of variance in the first principal component defined as follows:

$$
v=\frac{\lambda_{1}}{\sum_{n=1}^{N} \lambda_{n}}=\frac{\lambda_{1}}{N}
$$

where $\lambda_{n}$ is the $n$-th eigenvalue of the correlation matrix $Q$ sorted in descending order, i.e.,

$$
\lambda_{1}>\lambda_{2}>\ldots>\lambda_{N}
$$

Note that we have used $\sum_{n=1}^{N} \lambda_{n}=N$ because $\operatorname{Tr}(Q)=\sum_{n=1}^{N} Q_{i i}=N$ is invariant under a similarity transformation. This quantity $v$ in Eq. (6) can be used to estimate the effective 
dimensionality $d_{\mathrm{eff}}$ of the given data for the following reason: Formally speaking, $d_{\mathrm{eff}}$ is commonly estimated by selecting the smallest number of principal axes up to which the cumulative fraction of variance exceeds a certain level $\Lambda$ somewhere between $70-90 \%$ [24, 25]. Combining this method with the eigenvalue structure [Eq. (7)], we see that

$$
\frac{\sum_{n=1}^{d_{\mathrm{eff}}-1} \lambda_{n}}{N}<\Lambda<\frac{\sum_{n=1}^{d_{\mathrm{eff}}} \lambda_{n}}{N}<\frac{\lambda_{1} d_{\mathrm{eff}}}{N}=v d_{\mathrm{eff}} .
$$

In this sense, we argue that $\Lambda / v$ provides a lower bound for $d_{\text {eff. }}$ If $v \sim O(1)$, for example, the correlation is nearly one-dimensional, or we can say that it is "thin", and the first principal axis explains most of the variance in the data. If $v \ll 1$, on the other hand, the correlation structure has high effective dimensionality that cannot be reduced adequately along a one-dimensional axis; hence, it is "thick".

For polarization to be a meaningful description of party politics, the lawmakers need to occupy two diametrically opposite sides of the political landscape, making every issue nearly one-dimensional. In other words, politics can be diagnosed with polarization when both $b$ and $v$ are high in terms of the PCA results. More specifically, we set the criterion as $b$ greater than 5/9, the borderline between unimodal and bimodal distributions, in addition to $v \gtrsim 0.3$ so as to drop the lower bound of $d_{\text {eff }}$ down to $O(1)$ [26].

\section{RESULT}

With the MVH as a working hypothesis, let us look at the political polarization in the National Assembly of the Republic of Korea for the past 70 years. The whole period can be divided into three parts: The first corresponds to the First Republic, which was ended in 1960 by the April Revolution against autocracy. Shortly after the revolution, Korea experienced military dictatorships from 1961 to 1987. Opposition parties did exist, but they were always minorities by the rules of the game. The last period started in 1987 with the establishment of the Sixth Republic, after which Korea has been on the road to democracy. We will mainly contrast the last period (1988 - present) with the second under military governments $(1961-1987)$ because they are similar in length but substantially different in the political ideas.

Our question is how the different political structures have affected the degree of polarization, as measured by lawmakers' correlation in their co-sponsorship of bills [20, 27 34]: 


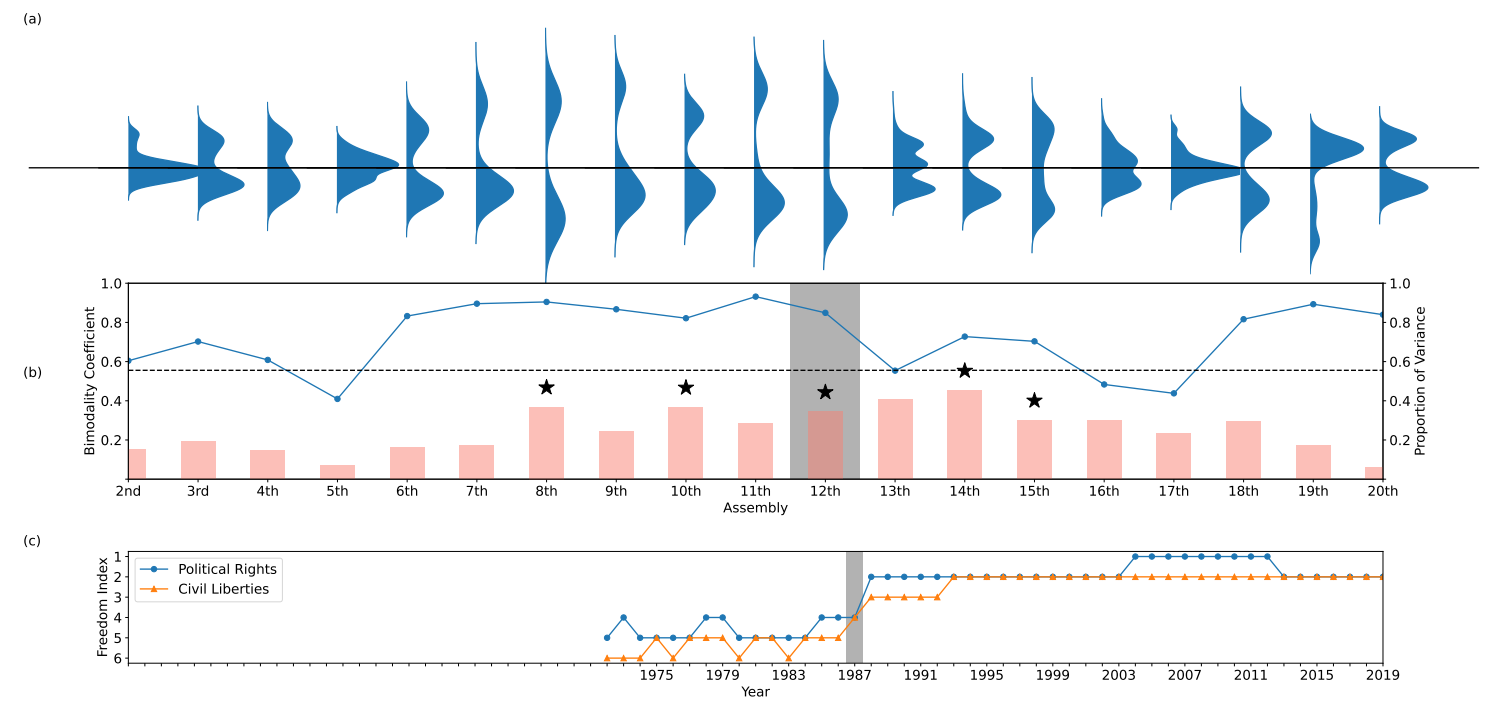

FIG. 1. Time evolution of political polarization in the National Assembly of the Republic of Korea. (a) Each year's distribution of lawmakers along the first principal axis. When two sessions exist in the same year because of an election, they are treated separately. When the distribution for an Assembly is bimodal, the clusters are almost always divided by party membership. (b) Sample bimodality coefficients [Eq. (5)] depicted as the line graph. The dashed horizontal line is for $\beta=5 / 9$ of the uniform distribution, which serves as a borderline between unimodal and bimodal distributions, and the bar graph shows the proportion of variance in the first principal component, denoted by $v$ [Eq. [6] ]. We have drawn asterisks when $b>5 / 9$ and $v>0.3$, and the shaded rectangle indicates the establishment of the Sixth Republic in 1987. (c) Political rights and civil liberties in Korea, according to Freedom in the World. Again, we have drawn a shaded rectangle to indicate the year 1987.

According to the MVH, it will decrease after democratization whereas we would have no reason to expect such an effect under a dictatorship. Our analysis indeed shows that the lack of democracy induced a high level of polarization, which in turn shrank the political landscape to nearly one dimension: Figure 1(a) shows each year's distribution of lawmakers along the first principal axis, and the corresponding bimodality coefficient $b$ [Eq. (5)], depicted as a line graph in Fig. 1(b), remained high under military governments from the early 1960s to the late 1980s. Consistent with the MVH, the accumulated tension was first released in the 
late 1980s when democracy began to work in the Sixth Republic, so the bimodality showed a meaningful decrease in the 13th Assembly (1988 - 1992), which was a period of transition from military dictatorship to formal democracy [Fig. 1(c)]. Thereafter, the line graph of $b$ shows wild fluctuations, but the difference from the earlier period is striking.

Another notable feature is that the proportion of variance explained by the first principal component, denoted by $v$ (the bar graph), had a clearly increasing tendency from the beginning of the dictatorship [see the 5th Assembly (1960 - 1963) in Fig. 11(b)] and eventually went beyond 50\% around the transition period in the early 1990s, which implies that the effective political dimension was close to one. Since then, the bar graph has decreased down to $10 \%$, which means a considerable increase in the effective dimensionality. In other words, politics has gradually become higher dimensional over the past 30 years. We have performed the permutation test for the time series of $v$ from the 14th Assembly, and the resulting $p$-value is less than $1 \%$.

\section{DISCUSSION}

When parties take over control over the government through elections, taking extreme positions across the political spectrum becomes risky for them. On the other hand, a dictatorship would hardly feel motivated to compromise with others' opinions: To evade such pressure towards the median systematically, military dictatorships abused the mediumconstituency system in the 1970s and 1980s, and some lawmakers were even awarded through recommendation of the president (Table II). The result was a high level of political polarization without relaxation for decades.

The establishment of the Sixth Republic in 1987 was a milestone in the political history of Korea, which allowed party politics to revive after a long military dictatorship. The government party failed to win a majority in the 1988 legislative election for the 13th Assembly, for the first time since 1950, and the government party had to merge with two opposition parties in 1990 to regain the majority. Since 1988, the degree of political polarization has fluctuated widely, and this should not be surprising if we consider that parties obtain power by socializing conflict and representing different views on it. Exactly which factor determines the varying degrees of polarization is unclear: For example, the bimodality in the 16th and the 17th Assemblies is unexpectedly low although they were typically marked by 
(a)

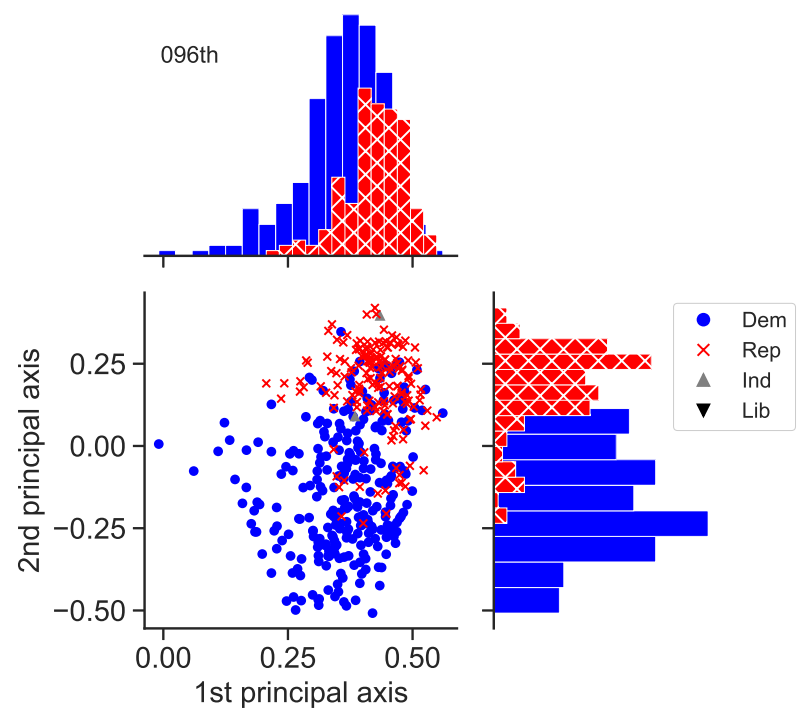

(b)

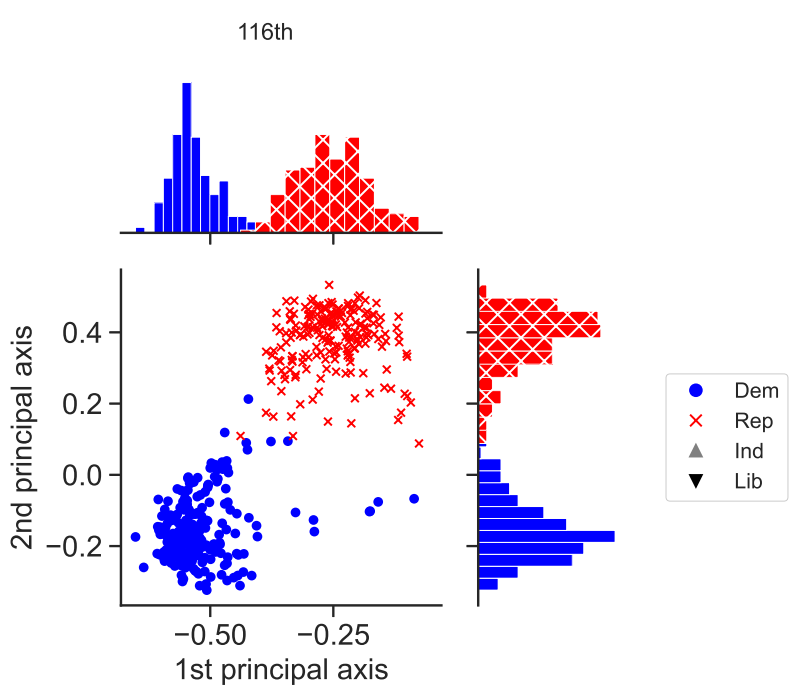

FIG. 2. Two-dimensional representation of co-sponsorship in the United States House of Representatives through PCA. The blue, red, and black dots mean Democrats, Republicans, and independent lawmakers, respectively. (a) The 96th Congress (January 15, 1979 - December 16, 1980) showed a considerable overlap between Democrats and Republicans, which was actually a common pattern before 2018. (b) Recent segregation of Democrats and Republicans is clearly visible in the 116th Congress (January 3, 2019 - January 3, 2021). Still, the first principal axis contains only $20 \%$ of the total variance, and the second axis does less than $10 \%$.

serious conflicts including a violent clash over presidential impeachment in 2004.

A more noticeable trend is that the principal axis occupies less and less of the total variance as time goes by. Despite the rise in the bimodality coefficient in the last decade, its actual contribution to polarization is far less significant than it was in the mid-1980's. This observation implies that the current party politics in the Assembly can no longer be described adequately along a one-dimensional axis: It should rather be understood as a combination of multiple cleavages that are simultaneously in action, and the MVH ceases to be a realistic description at this stage.

In this respect, our point is that the MVH could be better supported by non-vanishing polarization in the absence of democracy, where the political spectrum becomes nearly onedimensional between despotism and democracy. On the other hand, the intrinsic dynamics of party politics, which the MVH intends to describe, will eventually undermine its assumption on one-dimensionality because parties are, by nature, apt to introduce new dimensions of 
TABLE II. The three phases of polarization and democracy proposed in this work. The first phase corresponds to the pre-democratic state of Korea before 1987. The current constitution, the Sixth Republic of Korea, is still in the second phase [see Fig. 1(b)]. The third phase describes the United States House of Representatives, especially before 2018.

\begin{tabular}{cccc}
\hline phase dimensionality & bimodality & description \\
\hline I & close to one & high & pre-democratic \\
II & increasing & fluctuating & democratization \\
III & high & low & mature democracy \\
\hline
\end{tabular}

conflict into the public sphere. If we look at the United States House of Representatives as an example of a mature political system (Fig. 2), even today, for which the bimodality coefficient $b \gtrsim 0.7$ signals a record-high level of polarization, the political space of the United States is still far from one-dimensional in the sense that $v \approx 20 \%$, which is actually a little smaller than the $25 \%$ in the mid-1980s.

Based on these observations, we propose three phases of polarization in the course of political development (Table II): In the first phase, where party politics is under the control of an autocratic government, every conflict is projected onto a single political axis and is usually intensified without relaxation, so the situation is easily reduced to "You are either with us, or against us." In the second phase, the tension is released by the action of party politics. At this stage, parties discover additional dimensions of conflict, whereby the boundary between 'with us' and 'against us' gets blurred, which may lead to frequent changes in the political alignment of parties. Finally, in the last phase, mature party politics secures enough thickness to absorb conflicts and maintain social integration. Note that the one-dimensional party dynamics as assumed in the MVH holds true only in a very narrow region between the first and the second phases.

Admittedly, such a three-phase picture is a largely speculative one, calling for more empirical verification. Also, from the theoretical perspective, we are not dealing with the MVH in its rigorous sense: For example, the distinction between the median and the mean might be virtually nonexistent in political terms such as moderates, and empirically comparing the convergence point of lawmakers with the actual political position of the median voter among the electorate would be difficult. Our goal was rather to capture the nontrivial dynamics 
of political convergence and polarization by looking at the co-sponsorship data from the perspective of the MVH.

\section{SUMMARY}

In summary, we investigated the time evolution of political polarization in the National Assembly of Korea from 1950 to 2019 by applying PCA to co-sponsorship data. The degree of polarization was measured by using the bimodality coefficient of the distribution of lawmakers along the first principal axis, and we checked the proportion of variance explained by the first principal component. For the period of dictatorship from the early 1960s to the late 1980s, we observe no relaxation of political polarization, and the proportion of variance in the first principal component accumulated over time throughout this period. The political polarization was greatly reduced by the establishment of formal democracy in 1987. Since then, the level of polarization has fluctuated, but the proportion of variance in the first principal component has constantly decreased. The additional analysis of the United States House of Representatives suggests that both of these observables will eventually stay at low values in a mature democracy. Our observation provides a dynamic view of party politics in terms of the socialization of conflict, a noisy, yet inevitable, process of democracy.

\section{ACKNOWLEDGMENTS}

This work was supported by a research grant of Pukyong National University (2020).

\section{DECLARATIONS}

Not applicable.

[1] E. E. Schattschneider, The Semisovereign People: A Realist's View of Democracy in America (Wadsworth Publishing Company, Boston, MA, 1975).

[2] D. Adamany, Am. Political Sci. Rev. , 1321 (1972).

[3] G. C. Layman, T. M. Carsey, and J. M. Horowitz, Annu. Rev. Polit. Sci. 9, 83 (2006). 
[4] J. E. Campbell, Polarized: Making sense of a divided America (Princeton University Press, Princeton, NJ, 2018).

[5] A. Downs, An Economic Theory of Democracy (Harper \& Row, New York, 1957).

[6] R. G. Holcombe, Public Choice 61, 115 (1989).

[7] J. L. Barr and O. A. Davis, South. Econ. J., 149 (1966).

[8] E. J. Mathis and C. E. Zech, Am. J. Econ. Sociol. 45, 403 (1986).

[9] G. K. Turnbull and S. S. Djoundourian, Public Choice 81, 223 (1994).

[10] B. Milanovic, Eur. J. Political Econ. 16, 367 (2000).

[11] H. Kasper, Public Choice 10, 1 (1971).

[12] W. A. McEachern, Natl. Tax J. , 129 (1978).

[13] T. Romer and H. Rosenthal, J. Public Econ. 12, 143 (1979).

[14] G. A. Boyne, Public Choice 53, 201 (1987).

[15] D. C. Mueller, Public Choice III (Cambridge University Press, New York, 2003).

[16] N. Canen, M. O. Jackson, and F. Trebbi, "Endogenous networks and legislative activity," (2021), available at SSRN 2823338.

[17] L. Harbridge, Is bipartisanship dead?: Policy agreement and agenda-setting in the House of Representatives (Cambridge University Press, New York, 2015).

[18] J. M. Curry and F. E. Lee, Perspectives on Politics 17, 47 (2019).

[19] C. Park and W. Jang, Korean J. Appl. Stat. 30, 403 (2017).

[20] S. K. Baek, J. Kim, S. S. Lee, W. S. Jo, and B. J. Kim, Physica A , 125178 (2020).

[21] N. McCarty, Polarization: What Everyone Needs to Know (Oxford University Press, New York, 2019).

[22] J. B. Freeman and R. Dale, Behav. Res. Methods 45, 83 (2013).

[23] R. Pfister, K. A. Schwarz, M. Janczyk, R. Dale, and J. Freeman, Front. Psychol. 4, 700 (2013).

[24] I. T. Jolliffe, Principal Component Analysis (Springer, 1986) pp. 93-94.

[25] M. E. Wall, L. Rocha, and A. Rechtsteiner, "Singular value decomposition and principal component analysis," in A Practical Approach to Microarray Data Analysis, edited by D. P. Berrar, W. Dubitzky, and M. Granzow (Springer, 2002) Chap. 5, pp. 91-109.

[26] R. A. Serway and J. W. Jewett, Jr., Physics for Scientists and Engineers with Modern Physics (Brooks/Cole, Boston, MA, 2014) p. 10. 
[27] J. H. Fowler, Political Anal. 14, 456 (2006).

[28] M. A. Porter, P. J. Mucha, M. E. Newman, and A. J. Friend, Physica A 386, 414 (2007).

[29] Y. Zhang, A. J. Friend, A. L. Traud, M. A. Porter, J. H. Fowler, and P. J. Mucha, Physica A 387, 1705 (2008).

[30] B. M. Harward and K. W. Moffett, Legis. Stud. Q. 35, 117 (2010).

[31] K. T. Macon, P. J. Mucha, and M. A. Porter, Physica A 391, 343 (2012).

[32] M. P. Rombach, M. A. Porter, J. H. Fowler, and P. J. Mucha, SIAM J. Appl. Math. 74, 167 (2014).

[33] C. Andris, D. Lee, M. J. Hamilton, M. Martino, C. E. Gunning, and J. A. Selden, PloS one 10, e0123507 (2015).

[34] T. Colliri and L. Zhao, Sci. Rep. 9, 1 (2019). 